\title{
Catastrophic optical mirror damage in diode lasers monitored during single-pulse operation
}

Zegler, M.; Tomm, J.W.; Reeber, D.; Elsaesser, T.; Zeimer, U.; Larsen, Henning Engelbrecht; Petersen, Paul Michael; Andersen, Peter E.

Published in:

Applied Physics Letters

Link to article, DOI:

$10.1063 / 1.3133339$

Publication date:

2009

Document Version

Publisher's PDF, also known as Version of record

Link back to DTU Orbit

Citation (APA):

Zegler, M., Tomm, J. W., Reeber, D., Elsaesser, T., Zeimer, U., Larsen, H. E., Petersen, P. M., \& Andersen, P. E. (2009). Catastrophic optical mirror damage in diode lasers monitored during single-pulse operation. Applied Physics Letters, 94(19), 191101. https://doi.org/10.1063/1.3133339

\section{General rights}

Copyright and moral rights for the publications made accessible in the public portal are retained by the authors and/or other copyright owners and it is a condition of accessing publications that users recognise and abide by the legal requirements associated with these rights.

- Users may download and print one copy of any publication from the public portal for the purpose of private study or research.

- You may not further distribute the material or use it for any profit-making activity or commercial gain

- You may freely distribute the URL identifying the publication in the public portal 


\title{
Catastrophic optical mirror damage in diode lasers monitored during single-pulse operation
}

\author{
Mathias Ziegler, ${ }^{1, a)}$ Jens W. Tomm, ${ }^{1}$ David Reeber, ${ }^{1}$ Thomas Elsaesser, ${ }^{1}$ Ute Zeimer, ${ }^{2}$ \\ Henning E. Larsen, ${ }^{3}$ Paul M. Petersen, ${ }^{3}$ and Peter E. Andersen ${ }^{3}$ \\ ${ }_{1}^{1}$ Max-Born-Institut, Max-Born-Str. 2 A, 12489 Berlin, Germany \\ ${ }^{2}$ Ferdinand-Braun-Institut für Höchstfrequenztechnik, Gustav-Kirchhoff-Str. 4, 12489 Berlin, Germany \\ ${ }^{3}$ Department of Photonics Engineering, DTU Fotonik, Technical University of Denmark, \\ Risф campus Building 128, P.O. Box 49, 4000 Roskilde, Denmark
}

(Received 7 April 2009; accepted 21 April 2009; published online 11 May 2009)

Catastrophic optical mirror damage (COMD) is analyzed for $808 \mathrm{~nm}$ emitting diode lasers in single-pulse operation in order to separate facet degradation from subsequent degradation processes. During each pulse, nearfield and thermal images are monitored. A temporal resolution better than $7 \mu \mathrm{s}$ is achieved. The thermal runaway process is unambiguously related to the occurrence of a "thermal flash." A one-by-one correlation between nearfield, thermal flash, thermal runaway, and structural damage is observed. The single-pulse excitation technique allows for controlling the propagation of the structural damage into the cavity. We propose this technique for the analysis of early stages of COMD. (c) 2009 American Institute of Physics. [DOI: 10.1063/1.3133339]

Catastrophic optical mirror damage (COMD) represents a sudden degradation mechanism of semiconductor lasers and has been addressed in numerous papers. ${ }^{1-5}$ The present understanding of COMD may be summarized as follows.

- COMD affects predominantly, but not exclusively, the front facet of edge-emitting diode lasers if operated at elevated output powers.

- The status of the surface, quantified, e.g., by the surface recombination velocity, is a major extrinsic parameter that influences the COMD behavior, such as the COMD threshold. ${ }^{2,3}$

- The ultimate facet alteration process is believed to be induced by a thermal runaway process that can be described as a thermal microexplosion. ${ }^{1-5}$ Once going beyond a critical current, this process takes place with a typical delay of $\sim 100$ ns and with an even shorter duration. ${ }^{2,5}$

- In many reports, "dark bands" ${ }^{, 6,7}$ are described, which start at the facet and extend along the cavity, and even their formation has been detected by silicon charge-coupled device ( $\mathrm{Si}-\mathrm{CCD})$ cameras as moving bright spots. ${ }^{8,9}$

All these studies were, however, carried out by analyzing different types of devices under different operation conditions. Therefore, there exists substantial ambiguity and it is even still unclear whether the course of events along the runaway process is similar in all types of diode lasers. Addressing this issue becomes even more complicated as there are devices not showing any signs of COMD.

In this letter, we present a methodology that aims at establishing experimental evidence that links COMD for different types of devices and operation conditions. The key concept is to perform COMD experiments on a set of wellanalyzed standard devices, whose main failure mode is not COMD. A thermocamera is used for the unambiguous detection of the thermal runaway by the "thermal flash."10 Finally, we use single-pulse excitation in order to initiate the COMD,

${ }^{a)}$ Electronic mail: mziegler@mbi-berlin.de. but to avoid extensive subsequent degradation. Furthermore, additional motivation for this work arises from the desire to push the temporal resolution of thermographic COMD analysis from the millisecond to microsecond time scale. ${ }^{10}$

We investigate commercial $808 \mathrm{~nm}$ emitting high-power broad-area diode lasers, specified for reliable continuous wave (cw) operation up to $2 \mathrm{~W}$ with a compressively strained InAlGaAs single quantum well $(\mathrm{QW})$ embedded into a $\mathrm{Ga}_{0.4} \mathrm{Al}_{0.6} \mathrm{As}$ waveguide and $\mathrm{Ga}_{0.7} \mathrm{Al}_{0.3} \mathrm{As}$ claddings. ${ }^{11}$ The large optical cavity emitter with a stripe width of $200 \mu \mathrm{m}$ has chip dimensions of $1200 \times 400 \times 115 \mu \mathrm{m}^{3}$ (length $\times$ width $\times$ height). The facets are passivated and coated with standard dielectric mirrors. This study includes results obtained using 20 devices from one single batch with a very homogeneous parameter spread: threshold currents are $563 \pm 2 \mathrm{~mA}$ and operation currents at $2 \mathrm{~W}$ are $2327 \pm 4 \mathrm{~mA}$; all at $25^{\circ} \mathrm{C}$. For clarity, we present data from three devices $\mathrm{A}, \mathrm{B}$, and $\mathrm{C}$.

The setup is based on the one described in Ref. 10. While the microscope with the Si-CCD camera that monitors the nearfield (NF) was left unchanged, we added an InSbbased thermocamera CEDIP Titanium $560 \mathrm{M}$ equipped with a microscope. Its integration time is set to 7 or $100 \mu$ s resulting in a temperature resolution of 20 and $1.4 \mathrm{~K}$, respectively. The camera detects radiation in the $3.4-5.5 \mu \mathrm{m}$ infrared (IR) wavelength region with a spatial resolution of $6 \mu \mathrm{m}$. Both cameras monitor the whole active region and are synchronized to a pulse generator (rise time from $10 \%$ to $90 \%$ is measured as $<6 \mathrm{~ns}$ ). All experiments are performed at stabilized heat-sink temperatures of $25^{\circ} \mathrm{C}$.

Scanning electron microscopy was performed twice. First, we inspected the front facets by secondary electron (SE) imaging (parameters: $10 \mathrm{keV}, 150 \mathrm{pA}$ ). The subsequent removal of the $n$ metallization and the GaAs substrate by grinding and wet chemical etching allowed us to analyze the devices from the top. Both plan-view SE images and panchromatic cathodoluminescence (CL) maps (parameters: 25 $\mathrm{keV}, 10 \mathrm{nA}$ ) of the remaining epilayer structures were taken 

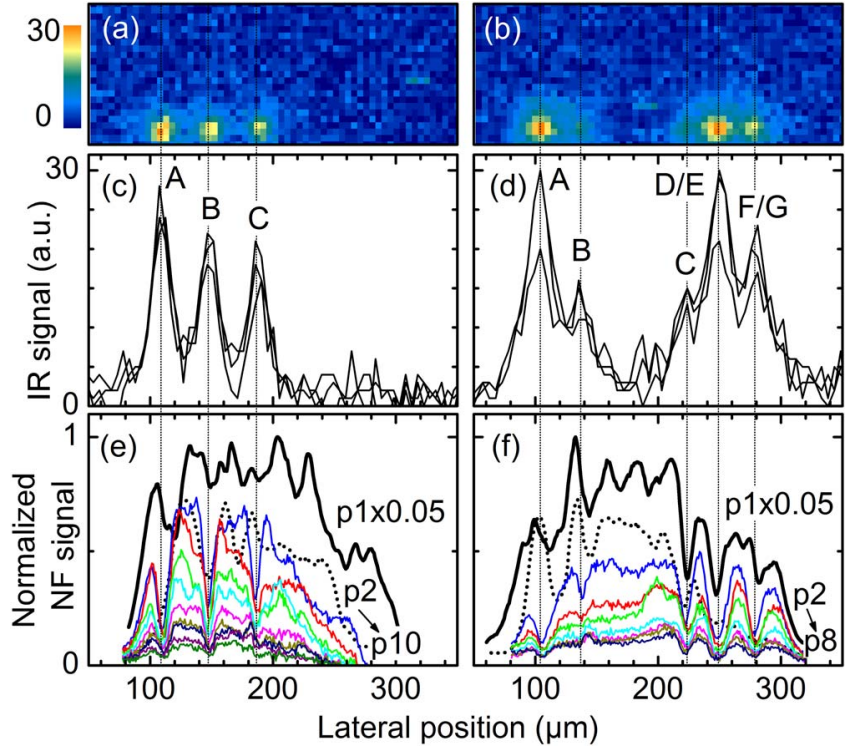

FIG. 1. (Color online) Data for 2- $\mu$ s-long 35 A current pulses. Left and right columns show results from devices A and B, respectively. [(a) and (b)] Thermal images and [(c) and (d)] corresponding lateral cuts through the three lines with maximal signal, with the homogeneous thermal background subtracted. The integration times were 100 and $7 \mu$ s for device A and B, respectively. [(e) and (f)] Normalized nearfield profiles (lateral). Profiles for the first current pulse (thick black lines) are scaled by 0.05 relative to the succeeding pulses (thin colored lines). Profiles at $2 \mathrm{~A}$, scaled by 0.05 as well, are given as dotted lines for comparison. The positions of the peaks in the thermal data and deep minima in the nearfield data agree (marked by dotted vertical lines and capital letters). The active stripe is located between 100 and $300 \mu \mathrm{m}$.

(temperature: $80 \mathrm{~K}$ ), where the latter represents mainly QW emission as proven by the CL spectra.

We started investigating the degradation behavior by stepwise increasing the single-pulse current (duration $2 \mu \mathrm{s}$ ), while monitoring NF and thermal images within each single pulse. At about four times the maximum recommended $\mathrm{cw}$ current (i.e., at $\sim 10$ A) "regular degradation"12 takes place. Although reliable detection of a thermal flash at this power level is possible, none was observed. New devices were used each time we increased the current in order to achieve similar starting conditions. We first detected thermal flashes at $35 \mathrm{~A}$; see Figs. 1(a)-1(f) for devices A and B. The data shows clear spatial correlations between distinct features related to the COMD; see lines and labels in all figures:

- Pronounced thermal flashes are detected as singular events that occur during the first current pulse only [Figs. $1(\mathrm{a})-1(\mathrm{~d})]$.

- A strong decrease of the NF signal by one to two orders of magnitude for subsequent pulses is observed and the NF profiles exhibit distinct deep minima exactly at the thermal flash positions [Figs. 1(e) and 1(f)]. Also at lower currents (15-20 A), the NF intensity is dramatically reduced after the first pulse due to regular degradation, while the thermal flash is absent (although it would be detectable).

- Front facet SE imaging (Fig. 2) shows extrusions only at 35 A. The locations exactly match the positions of the observed thermal flashes and even the sizes of these extrusions correspond well to the magnitude of those flashes (inset in Fig. 2). Notice that the extrusions at $F$ and $G$ are spaced by approximately $5 \mu \mathrm{m}$, which is below the IR-

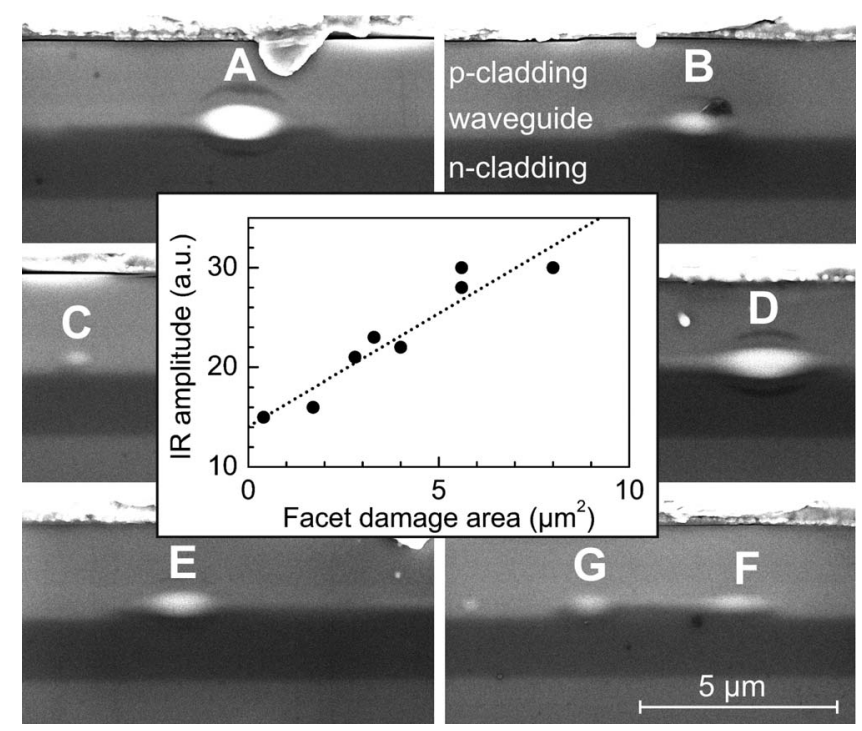

FIG. 2. SE images acquired from the front facet ( $p$-side up) of device B The epitaxial structure is indicated. Extrusions are found at exactly those positions marked in Fig. 1. Partial darkening of the $n$-type waveguide at these positions is visible as well. The inset shows the amplitude of the thermal flash vs the area of the COMD damage sites for devices A and B.

camera spatial resolution of nominally $\sim 6 \mu \mathrm{m}$.

- Results of destructive analysis after removal of the substrate by CL imaging are presented in Fig. 3. Starting at the front facets, we find very pronounced but short dark bands spreading into the cavity for about 3-8 $\mu \mathrm{m}$; also located at the thermal flashes positions. The striped CL pattern [even for the pristine reference sample C; see Fig. 3(c)] is caused by the patterned injection geometry of this laser structure, which involves 20 substripes within the broad area emitter stripe.
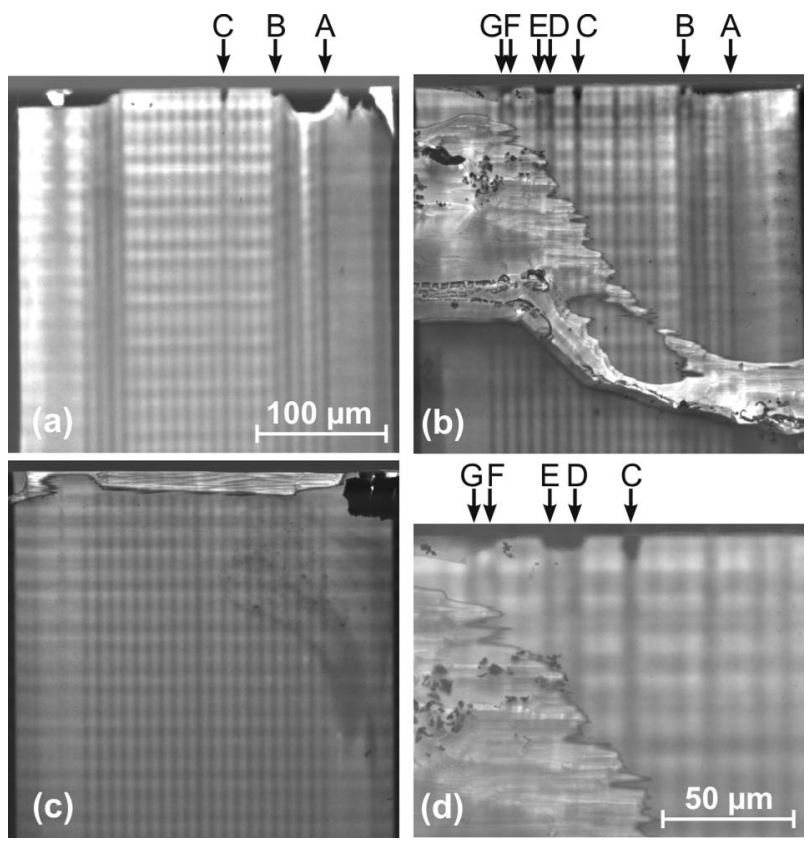

FIG. 3. Panchromatic CL images of the opened active regions (front facet up) of (a) device A, (b) device B, (c) pristine reference device C, and (d) device $\mathrm{B}$ at an expanded scale. Front facet damages are indicated by arrows and labeled according to Figs. 1 and 2. In [(b) and (d)], incomplete etching through the substrate resulted in partial covering of the active-region CL signal. 
Analysis of the different COMD signatures provides a number of insights. First, the ratios of the NF intensities during failure at $35 \mathrm{~A}$ and regular operation at $2 \mathrm{~A}$ [Figs. 1(e) and 1(f)] allow for an estimation of the duration of the thermal flash. Assuming a linear dependence of the NF intensity $I_{\mathrm{NF}}$ on current above threshold, we expect a numerical value of $I_{\mathrm{NF}}(35 \mathrm{~A}) / I_{\mathrm{NF}}(2 \mathrm{~A})=22 \pm 3$. In contrast, the measured ratio is $1.8 \pm 0.2$. Both values are, however, compatible if we assume regular operation only during an initial period of $\sim 100-200$ ns (i.e., 5\%-10\% pulse width) before the critical facet temperature is reached. Such temporal range is in good agreement with theoretical models. ${ }^{1,2,5}$ After this period, the thermal runaway occurs on a much faster 1 to $10 \mathrm{~ns}$ time scale, eventually resulting in COMD. ${ }^{2,4,5}$ During the rest of the pulse (i.e., $1.8-1.9 \mu \mathrm{s}$ ) the output power drops by a factor of $(22 \pm 3) \times(25 \pm 5) \approx(6 \pm 2) \times 10^{2}$, where the second factor is extracted from Figs. 1(e) and 1(f) as the apparent NF drop between the first two 35 A pulses, provided by the fact that the subsequent NF decrease is much weaker. Peak temperatures of $260-320{ }^{\circ} \mathrm{C}$ can be extrapolated ${ }^{13}$ from the raw thermographic data for this heating period. Also the very similar magnitudes of the thermal flashes recorded for device A at integration time of $100 \mu$ s [Figs. 1(a) and $1(\mathrm{c})]$ and for device B at integration time of $7 \mu \mathrm{s}$ [Figs. 1(b) and 1(d)] support that the flash-creating heating lasts for only such period. The temperature values are, however, only lower limits of much higher values as high as the materials melting points $\left(>1200{ }^{\circ} \mathrm{C}\right)$ due to spatial and temporal averaging as discussed in Ref. 10. It should be noted that competing temperature-probing techniques such as Raman and thermoreflectance spectroscopy, which have a higher spatial resolution do not allow for probing the occurrence of the thermal runaway during subsequent pulses since they are disturbed by the structural alteration of the facet during the first pulse. ${ }^{14}$

We also find signatures of propagating melt fronts as reported for other IR emitting devices ${ }^{8,9}$ as 3-8 $\mu \mathrm{m}$ long dark bands seen in the CL maps directly at the front facets [Fig. 3(d)]. The propagation velocities during the $1.8-1.9 \mu$ s heating period are calculated as $1-5 \mathrm{~m} / \mathrm{s}$, in accordance with Refs. 15 and 16. Starting from these pronounced dark bands, additional slightly "grayer bands" (Fig. 3) extend into the cavity. It is very likely that these weak bands are created upon completion of the thermal-runawayinduced damage of the first pulse. None of that is found for devices that failed at currents lower than $35 \mathrm{~A}$.

This means that the observed thermal flash is indicative for a catastrophic damage that is induced by a thermal runaway and that such process occurs only once and only at the highest currents applied in this study. Such behavior seemingly contrasts other studies on pulsed operated IR broad area devices, where successive propagation of melt fronts in subsequent pulses is assumed. ${ }^{16,17}$ Our findings, however, do not contradict such successive propagation, because the regular degradation ${ }^{12}$ of our devices does not involve front facet COMD as in Refs. 16 and 17. Nevertheless, we are able to artificially create the COMD at very high currents in our devices, because it is the fastest process. A sufficiently high temperature at the front facet provokes thermal runaway. The heat for this temperature increase is mainly produced through reabsorbed stimulated emission at the facet, which delivers energy from along the whole cavity. Heating at this surface happens on a faster time scale than any other heating effect and, consequently, the regular degradation ${ }^{12}$ follows temporally after the COMD. Apparently, these slower processes (that can be induced already at much lower currents than the COMD threshold) damage the device in a way that a new onset of thermal runaway is suppressed during subsequent pulses.

In summary, we reported on COMD experiments with diode lasers in single-pulse operation. During each pulse, we monitored both NF and thermal images and were able to unambiguously relate the onset of the thermal runaway process to the occurrence of a thermal flash. We detected such flashes with $<7 \mu$ s temporal resolution which is more than two orders of magnitude faster than previously reported. ${ }^{10}$ We also found them to clearly correlate with structural damages starting at the front facet. We demonstrated a one-byone correlation between strong NF minimum, thermal flash, thermal runaway, and structural damage. As a consequence of the single-pulse-excitation technique, the propagation of dark bands ${ }^{6}$ from the front facet is halted after the first pulse. Finally, because of the rapidness of the thermal runaway, we propose the described technique for the test of the facet stability and the intentional preparation of early stages of COMD; even for diode lasers that regularly fail by mechanisms other than COMD.

The authors thank Helen Lawrenz for expert technical assistance. Funding by the European Commission within the project WWW.BRIGHTER.EU under Contract No. IST2005-035266 is acknowledged.

${ }^{1}$ C. H. Henry, P. M. Petroff, R. A. Logan, and F. R. Meritt, J. Appl. Phys. 50, 3721 (1979).

${ }^{2}$ W. Nakwaski, J. Appl. Phys. 57, 2424 (1985).

${ }^{3}$ R. Schatz and C. G. Bethea, J. Appl. Phys. 76, 2509 (1994).

${ }^{4}$ P. G. Eliseev, Reliability Problems of Semiconductor Lasers (Nova Science, Commack, 1991), p. 213.

${ }^{5}$ W. R. Smith, J. Appl. Phys. 87, 8276 (2000).

${ }^{6}$ With the term dark bands, we denote lines of reduced QW emission as detected in many studies by micro-PL or CL mapping of the active area plane (after removal of contacts and sometimes substrate). Typically these lines propagate from a starting point (mostly) at the facet into the bulk along the propagation directions of the optical mode(s).

${ }^{7}$ M. Bou Sanayeh, A. Jaeger, W. Schmid, S. Tautz, P. Brick, K. Streubel, and G. Bacher, Appl. Phys. Lett. 89, 101111 (2006).

${ }^{8}$ K. H. Park, J. K. Lee, D. H. Jang, H. S. Cho, C. S. Park, K. E. Pyun, J. Y. Jeong, S. Nahm, and J. Jeong, Appl. Phys. Lett. 73, 2567 (1998).

${ }^{9}$ J. H. Jacob, R. Petr, M. A. Jaspan, S. D. Swartz, M. T. Knapczyk, A. M. Flusberg, A. K. Chin, and I. Smilanski, Proc. SPIE 7198, 719815 (2009).

${ }^{10}$ M. Ziegler, J. W. Tomm, T. Elsaesser, C. Matthiesen, M. Bou Sanayeh, and P. Brick, Appl. Phys. Lett. 92, 103514 (2008).

${ }^{11}$ Devices of type SPL CG81-2S have been purchased at http:// www.rutronik.com.

${ }^{12}$ Regular degradation of this type of devices involves gradual power loss and narrowing of the nearfield starting at the edges of the emitter stripes. It is thermally activated and does not involve the front facet.

${ }^{13}$ This data is obtained by using an extrapolated calibration curve that has been established from calibration measurements of a nonoperating device in the $25-100{ }^{\circ} \mathrm{C}$ range.

${ }^{14}$ T. J. Ochalski, D. Pierścińska, K. Pierściński, M. Bugajski, J. W. Tomm, T. Grunske, and A. Kozlowska, Appl. Phys. Lett. 89, 071104 (2006).

${ }^{15}$ B. W. Hakki and F. R. Nash, J. Appl. Phys. 45, 3907 (1974).

${ }^{16}$ O. Ueda, K. Wakao, S. Komiya, A. Yamaguchi, S. Isozumi, and I. Umebu, J. Appl. Phys. 58, 3996 (1985)

${ }^{17}$ C. W. Snyder, J. W. Lee, R. Hull, and R. A. Logan, Appl. Phys. Lett. 67, 488 (1995). 